\title{
Polymer anion-selective membranes for electrolytic splitting of water. Part II: Enhancement of ionic conductivity and performance under conditions of alkaline water electrolysis
}

\author{
Jaromír Hnát • Martin Paidar · Jan Schauer • \\ Jan Žitka $\cdot$ Karel Bouzek
}

Received: 20 February 2012/ Accepted: 5 May 2012/Published online: 22 May 2012

(C) Springer Science+Business Media B.V. 2012

\begin{abstract}
An attempt was made to increase the ionic conductivity of novel, heterogeneous, anion-selective membranes by increasing the porosity of their surface skin. This was based on the addition of a water-soluble component, namely poly(ethylene-ran-propylene glycol), to an inert polymer matrix, based on low-density polyethylene, while mixing it with the ion-exchange particles. A series of membranes was prepared, consisting of $66 \mathrm{wt} \%$ of anionexchange phase represented by a styrene-divinyl benzene copolymer matrix with quaternary ammonium functional groups and an inert polymer matrix in a mixture with variable amounts of water-soluble component added. The membranes were subsequently tested with respect to their morphology, mechanical properties, apparent ion-exchange capacity, ionic conductivity, and performance under conditions of alkaline water electrolysis. When added in the appropriate amount, the addition of a water-soluble component was found to improve the electrochemical properties of the resulting membrane efficiently, while at the same time not reducing its mechanical properties to below a critical level.
\end{abstract}

Keywords Water electrolysis - Alkaline environment . Polymer electrolyte . Conductivity enhancement . Water-soluble additive

J. Hnát · M. Paidar · K. Bouzek $(\bowtie)$

Department of Inorganic Technology, Institute of Chemical

Technology Prague, Technická 5, 16628 Prague 6,

Czech Republic

e-mail: bouzekk@vscht.cz

J. Schauer · J. Žitka

Institute of Macromolecular Chemistry, Academy of Sciences

of the Czech Republic, Heyrovsky Sq. 2, 16206 Prague,

Czech Republic

\section{Introduction}

In the first part of this study [1], the problem of alkaline electrolysis as the only recently established industrial water electrolysis technology was discussed in detail. This especially concerns its economic competitiveness with currently used technologies for fossil fuel-based hydrogen production and the technological challenges that still remain to be solved. These challenges are mainly connected with an increase in process efficiency and flexibility. Attention also focuses on the possibility of avoiding the use of dangerous chemicals, such as concentrated $\mathrm{KOH}$ solution. The motivation for this endeavor mainly consists in the search for sufficiently reliable, flexible, and easy to operate technology to stabilize the electricity distribution grid. This need was initiated by the rapid increase in the production capacity of the relatively unstable renewable energy sources connected to the grid [2, 3]. Hydrogen represents an attractive means of storing excess energy, which can be converted back into electricity when needed, e.g., by means of a fuel cell or combustion engine. It can also be used as a fuel or a raw material in various industrial processes, thus reducing their $\mathrm{CO}_{2}$ footprint. Of course, the latter statement only holds if hydrogen is produced from renewable sources.

Currently, several approaches to electrochemical water decomposition are being studied. Besides the alkaline route, the main alternative is proton-exchange membrane (PEM) water electrolysis. Recently, increasing attention has also been paid to high temperature water electrolysis utilizing a ceramic membrane as the electrolyte [4]. Whereas PEM technology is characterized by high process intensity and flexibility, the high temperature option benefits from high efficiency and replacement of part of the electrical energy needed to decompose water by cheaper 
(preferably waste) heat energy [4]. Neither of these technologies, however, has been sufficiently developed to justify their application on an industrial scale. In the case of the PEM process, the limitations are mainly related to the high demands of this technology on the platinum metal electrocatalysts. In the case of the high temperature process, serious material problems still hinder the wide use of this approach. Moreover, the latter technology is generally not flexible and it is intended to be used more for "leveling" the distribution grid in the medium to long term. The alkaline route is currently the only promising technology for broad practical application on a medium to large scale.

The relatively low efficiency and limited flexibility of the process are caused by the demands originally made of this technology. In the first place, this meant robustness. Efficiency was not the main requirement because this technology was typically used in locations with an excess of electricity production. This requirement resulted in the utilization of nickel-based, massive, two-dimensional electrodes, a thick diaphragm as an electrode compartment separator, and a highly concentrated $\mathrm{KOH}$ solution (typically $30 \mathrm{wt} \%$ ) as the electrolyte [5]. The high $\mathrm{KOH}$ concentration is used to reduce the voltage ohmic drop in the cell between the electrodes. Such a system is characterized by relatively low flexibility and insufficient efficiency $(55-76 \%)[5,6]$. A significant improvement in this situation is expected if the diaphragm and also, in an ideal case, the $\mathrm{KOH}$ in the electrolyte solution were to be replaced by an anion-selective polymer electrolyte. In time, this has to be connected with the utilization of porous, gas-evolving electrodes with a suitable electrocatalyst. This change currently represents an important challenge due to the relatively low stability of this type of material in a strongly alkaline environment at elevated temperatures [7].

The problem of identifying a suitable anion-selective polymer electrolyte is one topic of this series of studies. Attention is focused on heterogeneous polymer membranes. The motivation for this selection is the greater robustness of this group of materials. This is, however, attained at the expense of their ionic conductivity. In the first part of this study [1], attention is focused on the stability of the selected anion-selective functional groups in an alkaline environment at different temperatures and on the influence of the polymer binder on the electrochemical properties of the resulting heterogeneous anion-selective membranes. Besides the properties of the ion-exchange material, the polymer binder was also found to play an important role in the resulting behavior of the membrane. Apart from percolation of the individual ion-selective particles in the membrane structure, the surface skin film was found to play an important role. The skin film generally covering the surface of heterogeneous membranes is a result of a production procedure based on compression molding of the membrane from a mixture of melted matrix polymer and ion-exchange resin particles. The composition of the skin layer is generally different from that of the membrane core [8-10]. It typically consists exclusively of inert polymer binder which represents a barrier to ion transportation. A membrane with a linear polyethylene (LIPE) matrix showed the highest number of defects in the skin film covering the membrane surface. At the same time, this membrane showed the highest ionic conductivity and the best performance in the water electrolysis cell of the materials tested. Although the ionic conductivity of this membrane showed a satisfactory value of 5-6 S m $\mathrm{m}^{-1}$, depending on the temperature, its water electrolysis performance was not as good as expected. The target of the present study, representing the second part of this series, is to improve this behavior.

The membrane composition was optimized in the first part of this study [1]. The only way to further improve its performance is by artificially increasing the porosity of the surface skin film, which represents an important barrier to the transport of ions across the membrane. This effect is especially important under current load. In the present study, the surface skin layer was disturbed by the addition of a water-soluble component to the inert polymer matrix. It was then washed out of the membrane during its activation before application. Poly(ethylene-ran-propylene glycol) was selected as a suitable water-soluble addition. This polymer possesses the required compatibility with the matrix polymer, is easy to handle, and is quantitatively removed from the membrane structure during its activation. The heterogeneous membrane samples tested consisted of $66 \mathrm{wt} \%$ of anion-selective phase represented by a styrenedivinyl benzene copolymer matrix with quaternary ammonium functional groups, a variable amount of inert polymer matrix represented by low-density polyethylene (LDPE) and a variable amount of the water-soluble component. For this work, LDPE was selected instead of LIPE although the latter material showed the best properties in [1]. This was because its properties are appropriate for the desired application. This concerns namely its higher Vicat softening temperature $\left(93{ }^{\circ} \mathrm{C}\right)$ [11] compared to LIPE $\left(77^{\circ} \mathrm{C}\right)$ [12], allowing utilization of a membrane based on this matrix polymer at temperatures up to $90{ }^{\circ} \mathrm{C}$ without any danger of its mechanical collapse and also its high resistance to an alkaline environment [13]. Moreover, preliminary experiments showed that the addition of a water-soluble component resulted in equalization of the electrochemical properties of membranes based on LIPE and LDPE matrix polymers (results not shown here).

The activated membranes prepared in this study were characterized from the point of view of their morphology, mechanical properties, apparent ion-exchange capacity, ionic conductivity, and under conditions of alkaline water 
electrolysis. During alkaline water electrolysis, electrochemical impedance spectroscopy was used to evaluate the kinetic parameters of the cell.

\section{Experimental}

\subsection{Materials}

Low-density polyethylene (ExxonMobil ${ }^{\mathrm{TM}}$, LD 605BA) and a water-soluble additive poly(ethylene glycol-ranpropylene glycol) (PEG-PPG) (Sigma-Aldrich, average $\left.M_{n}=12,000\right)$ were used as received. Milled anion-selective particles Dowex Marathon A (Dow) with a predominant particle size of 10-30 $\mu \mathrm{m}$ and ion-exchange capacity (IEC) of $3.90 \mathrm{meq} \mathrm{g}^{-1}$ of dry particles were obtained from MEGA Co. (Czech Republic). As a reference, a commercially available anion-selective membrane A201 (developing code A006, Tokuyama Co., Japan) with IEC of 1.7 meq $\mathrm{g}^{-1}$ [14] was used.

\subsection{Membrane preparation}

Anion-selective particles in the $\mathrm{Cl}^{-}$cycle $(66 \mathrm{wt} \%)$ were blended with LDPE and a water-soluble additive $(0,1.7$, $3.4,6.8$, or $10.2 \mathrm{wt} \%$ ) in a Brabender Plasti-corder PLE 651 at $140{ }^{\circ} \mathrm{C}$ until the mixture became optically homogeneous and its torque value constant. The blend was pressmolded between two poly(ethylene terephthalate) films at $140{ }^{\circ} \mathrm{C}$ and at $10 \mathrm{MPa}$ for $3 \mathrm{~min}$ and then cooled to room temperature within $4 \mathrm{~min}$ to form a flat film. The resulting membranes were typically $0.30 \mathrm{~mm}$ thick.

\subsection{Pretreatment of the membranes}

Before characterization, the membranes have to be pretreated appropriately to remove the water-soluble component from their structure and to activate ion-exchange particles. The membrane activation used in this particular study proceeded in the following steps:

(1) Immersion in demineralized water for $24 \mathrm{~h}$.

(2) Immersion in $0.1 \mathrm{~mol} \mathrm{dm}^{-3}$ solution of $\mathrm{NaOH}$ for $2 \mathrm{~h}$.

(3) Immersion in $0.1 \mathrm{~mol} \mathrm{dm}^{-3}$ solution of $\mathrm{HCl}$ overnight.

(4) Immersion in $0.1 \mathrm{~mol} \mathrm{dm}^{-3}$ solution of $\mathrm{NaOH}$ for $4 \mathrm{~h}$.

(5) Immersion in demineralized water for $24 \mathrm{~h}$.

\subsection{Membrane morphology}

The morphology of the prepared anion-selective membranes was investigated using a Hitachi scanning electron microscope (SEM) S4700. Samples of the membranes were dried in a desiccator over molecular sieves. To obtain cross section images, a dry sample was immersed in liquid nitrogen and broken. Samples were coated with an $\mathrm{Au} / \mathrm{Pd}$ layer approximately 20 -nm thick by vacuum sputtering.

\subsection{Mechanical properties}

The mechanical properties of the membranes were tested using an Instron 3365 mechanical testing instrument. A sample of the tested membrane with defined dimensions was placed in a pneumatic holder. The samples were stretched by $5 \mathrm{~mm} \mathrm{~min}{ }^{-1}$ until tensile failure occurred. At the point of tensile failure, the values of tensile strength, tensile modulus, and energy to break were obtained.

\subsection{Apparent ion-exchange capacity}

The apparent ion-exchange capacity of the membrane is relevant to the conductivity of the membrane in the $\mathrm{OH}^{-}$ cycle. This characteristic was evaluated by potentiometry during the transition of the membrane sample from the $\mathrm{OH}^{-}$to the $\mathrm{Cl}^{-}$cycle. The potential response of a Ross combined glass electrode (Orion) was recorded during the experiment using a Keithley 6514 electrometer with high input impedance (200 T $\Omega$ ). The potential value of the glass electrode was converted into the concentration of displaced $\mathrm{OH}^{-}$ions in the solution by a calibration curve. This series of experiments was performed in $125 \mathrm{ml}$ of $0.1 \mathrm{~mol} \mathrm{dm}^{-3}$ $\mathrm{NaCl}$ solution in a gas-tight cell under argon atmosphere to avoid the effect of carbon dioxide. Decarbonized demineralized water was used in the experiments.

\subsection{Ionic conductivity (IC)}

The in-plane ionic conductivity of the membranes was determined in a tempered box in gas-tight cell in an environment of deionized water (i.e., $100 \%$ relative humidity) by means of electrochemical impedance spectroscopy (EIS) in a four-electrode arrangement. A Solartron SI1250 frequency response analyzer and Solartron SI1287 electrochemical interface were used to accomplish this task.

\subsection{Alkaline water electrolysis}

A single alkaline laboratory electrolyzer was used to test the membranes' performance under alkaline water electrolysis (AWE) conditions. Ni foam electrodes with an active area of $2 \times 2 \mathrm{~cm}^{2}$ were attached directly to the membrane surface. The rear side of the electrodes was washed by a $10 \mathrm{wt} \% \mathrm{KOH}$ solution at a set temperature. The performance of the cell was tested within a cell voltage range of 1.5-2.0 V. To evaluate membrane resistance during the process of alkaline water electrolysis, EIS was 
used in a two-electrode arrangement with the same equipment as described in chapter 2.7. A frequency range of $10 \mathrm{kHz}-100 \mathrm{~Hz}$ and a perturbation signal amplitude of $5 \mathrm{mV}$ were used.

\section{Results and discussion}

\subsection{Composition of the tested membranes}

All samples were prepared and tested in the form of heterogeneous anion-selective membranes. The individual components used were specified in the experimental part of this manuscript. The anion-exchange phase, represented by Dowex Marathon A anion-exchange resin particles, consisted of the styrene-divinyl benzene copolymer matrix with quaternary ammonium functional groups shown in Fig. 1. In the previous study, this functional group was evaluated to be the most stable one under conditions relevant to alkaline water electrolysis [1]. The prepared membranes always contained $66 \mathrm{wt} \%$ of the anion-selective phase. Inert polymer was the phase represented by pure LDPE mixed with PEG-PPG. The content of PEG-PPG was changed in the range of $0.0-10.2 \mathrm{wt} \%$ to test the influence of its content on the resulting membrane properties. Higher PEG-PPG contents were not tested as membranes based on a matrix polymer of such a composition lost their mechanical stability. The composition of the prepared and tested membranes is summarized in Table 1.

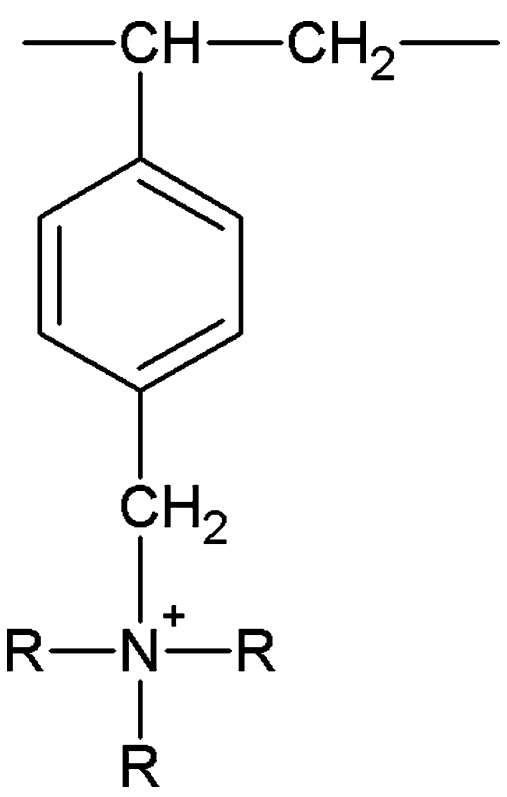

Fig. 1 Structure of the anion-selective functional group used in this study
Table 1 Composition of the tested heterogeneous anion-exchange membranes

\begin{tabular}{lll}
\hline $\begin{array}{l}\text { LDPE content } \\
\text { in wt } \%\end{array}$ & $\begin{array}{l}\text { PEG-PPG } \\
\text { content in wt } \%\end{array}$ & $\begin{array}{l}\text { Functional particle } \\
\text { content in wt } \%\end{array}$ \\
\hline 34.0 & 0.0 & 66.0 \\
32.3 & 1.7 & 66.0 \\
30.6 & 3.4 & 66.0 \\
27.2 & 6.8 & 66.0 \\
23.8 & 10.2 & 66.0 \\
\hline
\end{tabular}

\subsection{Membrane morphology}

As discussed above, the addition of PEG-PPG to the LDPE matrix is aimed to increase the porosity of the skin layer of the prepared heterogeneous membrane and thus to improve its performance in the water electrolysis process. This theory is based on the previous work reported in the first part of this series [1]. First, the impact of the addition of a water-soluble component on the morphology of the heterogeneous membranes prepared was closely examined. The development of the morphology of the surface skin layer of the activated heterogeneous membrane with the PEG-PPG content in the polymer matrix is documented in Fig. 2.

As clearly shown in these photographs, the addition of PEG-PPG to the matrix polymer clearly fulfills expectations. The number of defects in the skin layer significantly increased with its increasing content in the matrix polymer. Whereas for the addition of $3.4 \mathrm{wt} \%$ of water-soluble component, the increase in the membrane surface area covered by defects is still moderate at $10.2 \mathrm{wt} \%$ the area free of the skin layer becomes predominant. As already stated, an increase in the porosity of the skin film improves contact between the environment surrounding the membrane and its ionically conductive phase. It can thus significantly improve the conductivity of the membrane and especially its behavior under current load. On the other hand, too extensive opening of the skin film may have a negative impact on the membrane properties. This may negatively influence its electrochemical properties to a certain degree because of the loss of ion-exchanger particles which are no longer fixed in the membrane structure by the skin layer. Furthermore, the mechanical stability of the membrane may have deteriorated below an acceptable level.

It is to be expected that, besides the surface skin layer, the morphology of the membrane interior is also influenced by the PEG-PPG addition to the matrix polymer. SEM pictures of cross sections of the membranes with various amounts of water-soluble component are summarized in Fig. 3. Owing to the heterogeneous structure of the membranes, in these photographs, it is difficult to identify any changes in their morphology connected with the addition of 

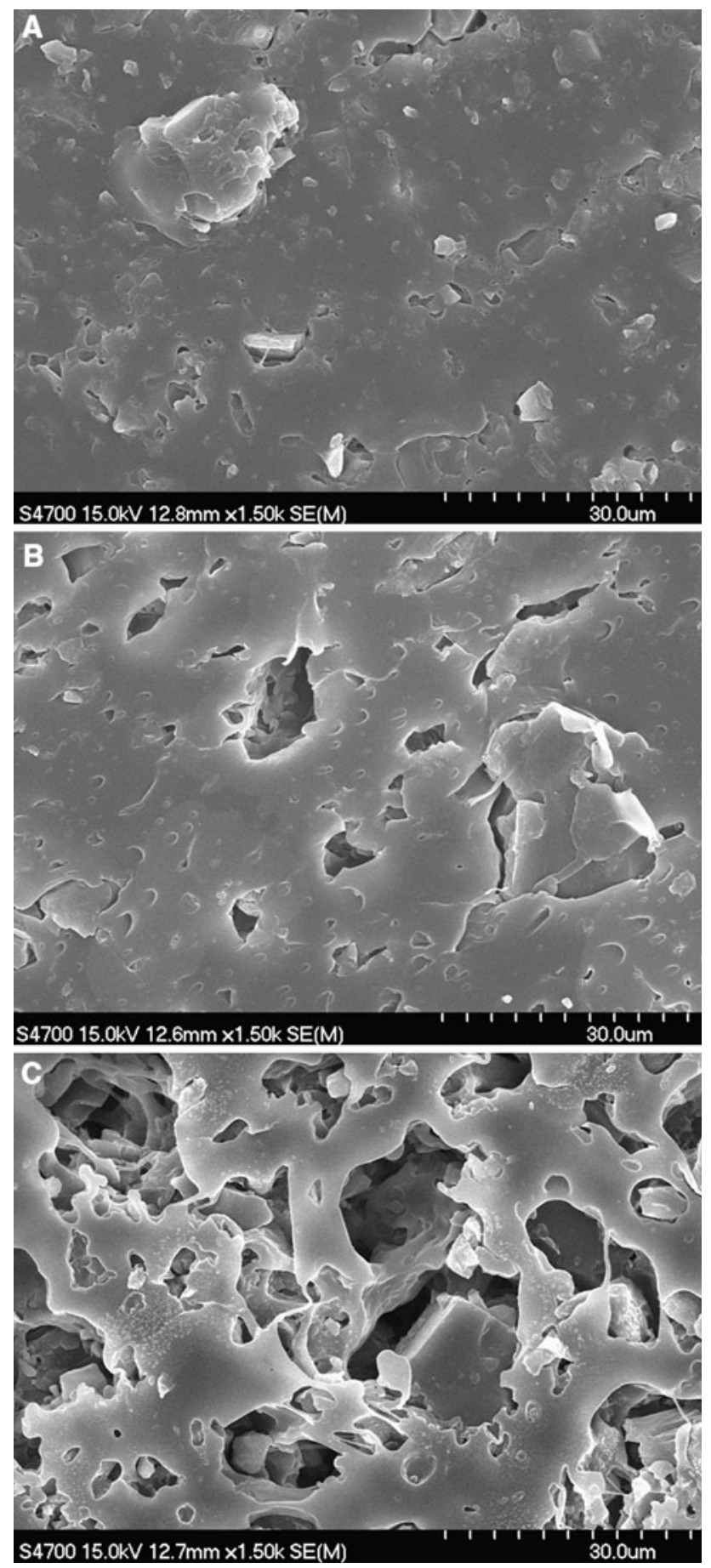

Fig. 2 SEM images of the surface skin layer of the activated anionselective heterogeneous membranes. a $0 \mathrm{wt} \%$ PEG-PPG, b $3.4 \mathrm{wt} \%$ PEG-PPG, c 10.2 wt \% PEG-PPG addition

the water-soluble component. A difference in the crosssectional morphology is only visible with the highest PEGPPG content in the membrane. The material also seems to be more porous in its interior; however, at this stage, the difference cannot be quantified. Moreover, these pores may act positively on the conductivity of the membrane.
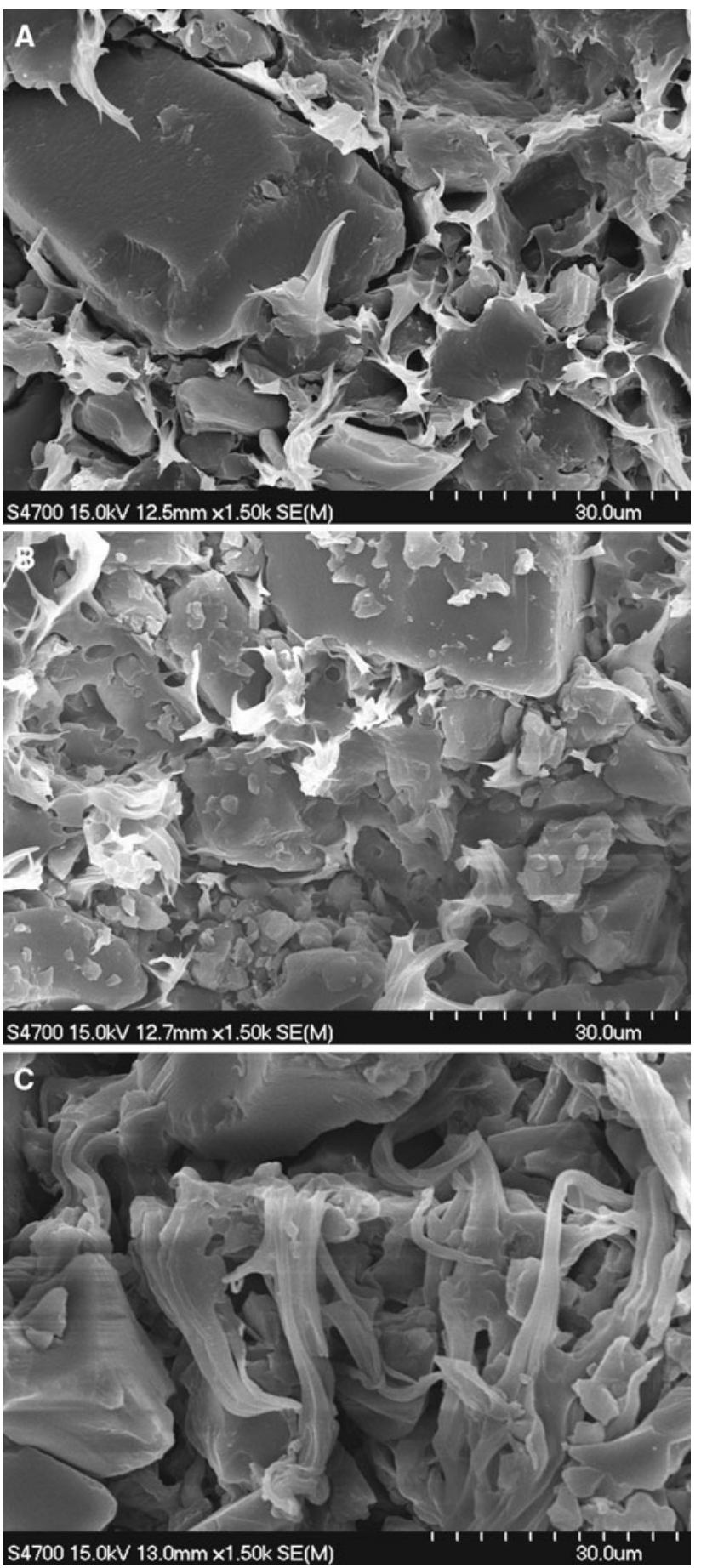

Fig. 3 SEM images of the cross section of the activated anionexchange membranes with: a $0.0 \mathrm{wt} \%$ PEG-PPG, b $3.4 \mathrm{wt} \%$ PEGPPG, and c 10.2 wt $\%$ PEG-PPG addition

However, in this case, too, an excessive increase in the porosity of the matrix polymer may cause the mechanical intactness of the membrane to be distorted. In such a case, the distance between the ion-selective particles will increase. Such behavior would have a negative impact on the membrane conductivity. 


\subsection{Mechanical properties}

As discussed in the previous chapter, the mechanical stability of the membrane is important not only from the point of view of the electrolysis cell construction, but also with respect to its electrochemical properties. In this study, the influence of the water-soluble addition on the membrane's tensile strength (Fig. 4a), tensile modulus (Fig. 4b), and energy necessary to break (Fig. 4c) was tested. As expected, in all cases, the mechanical properties rapidly decreased with increasing amounts of water-soluble component added to the structure of the membrane. This is clearly due to the decreasing content of the inert polymer phase which insures the mechanical stability of the membrane [15]. Nevertheless, even this deterioration is rather rapid, membranes with a water-soluble additive content up to $3.4 \mathrm{wt} \%$ seem to have promising properties. An activated membrane with this addition shows a decrease in tensile strength of only approximately $40 \%$ and energy to break even less significantly of $22 \%$ compared to the original membrane. The decrease in tensile modulus is more significant, but still satisfactory. These results have to be borne in mind for a final evaluation of the optimal water-soluble addition to the matrix polymer during membrane production.

\subsection{Apparent ion-exchange capacity}

This value represents the number of $\mathrm{OH}^{-}$ions exchanged from the unit of mass (or volume) of the membrane to the surrounding solution for suitable anions in the present case for $\mathrm{Cl}^{-}$. Owing to the $\mathrm{pH}$ equilibrium inside the membrane, this value does not correspond to the concentration of all ion-exchange sites inside the membrane. For a more detailed discussion of this phenomenon, see [1]. Nevertheless, this situation corresponds to the membrane in the alkaline water electrolysis operation because this process is based on the transportation of $\mathrm{OH}^{-}$through the electrolyte. For this reason, this was also followed in the present case. The results obtained for the series of membranes studied are summarized in Fig. 5.

From this figure, it follows that IEC is not significantly affected by the addition of PEG-PPG. A value of approximately $2 \mathrm{mmol} \mathrm{g}_{\text {dry }}^{-1}$ membrane was found for all membranes. The variation observed is within experimental error. A slight IEC increase for the membrane with an addition of $10.2 \mathrm{wt} \%$ of the water-soluble component is related to a reduction of the amount of inert phase in the membrane structure, not to essential changes in the membrane properties. Such behavior stems from the fact that the total amount of anion-exchange particles and functional groups was not primarily affected by the addition of the water-soluble component, see Table 1 . The second, more
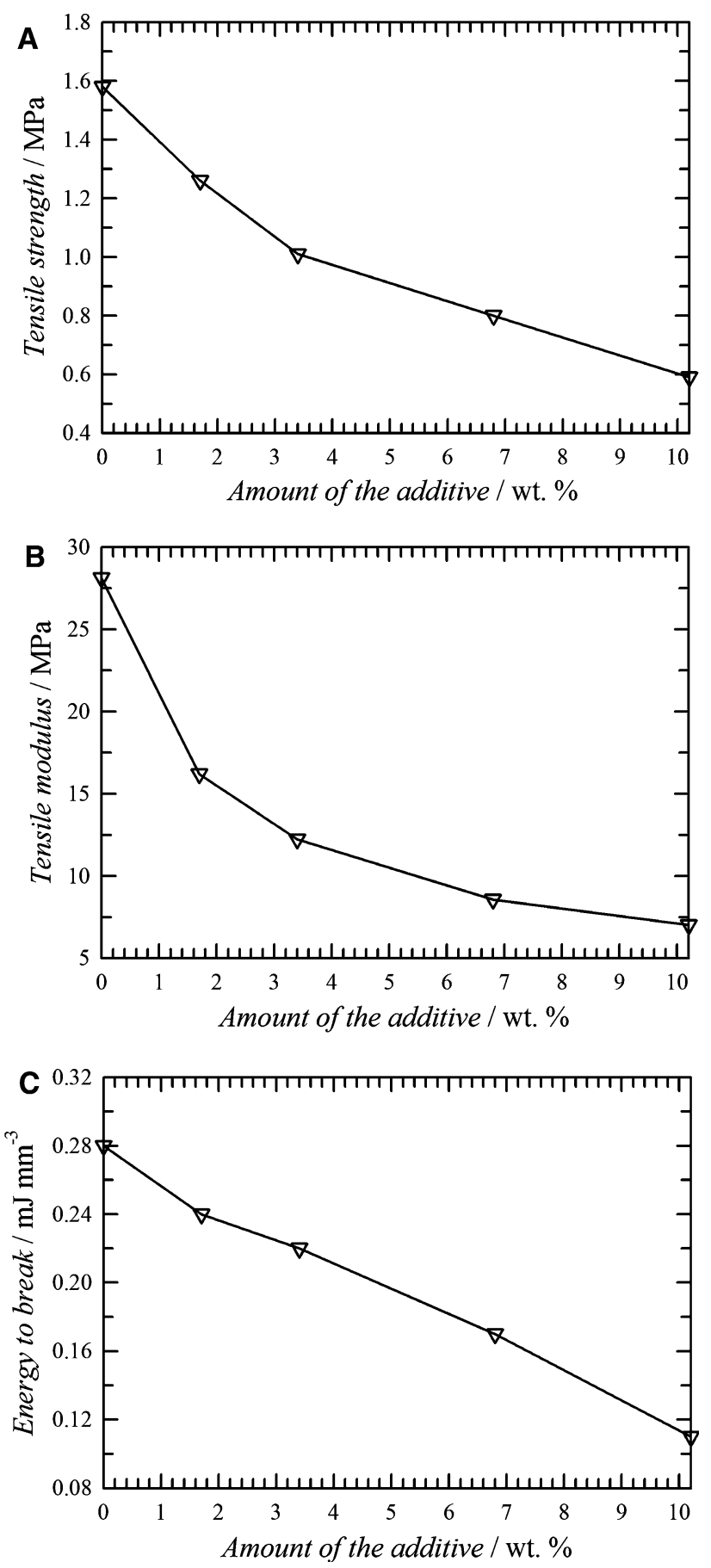

Fig. 4 Dependencies of a tensile strength, $\mathbf{b}$ tensile modulus, c energy to break of the activated membrane on the amount of water-soluble component added to the matrix polymer during production

important reason, is that the IEC measurement is an equilibrium one. The final amount of exchanged ions is not evaluated until the steady state is reached, i.e. all replaceable ions have been exchanged. The observed values of the IEC are comparable with those published in the literature 


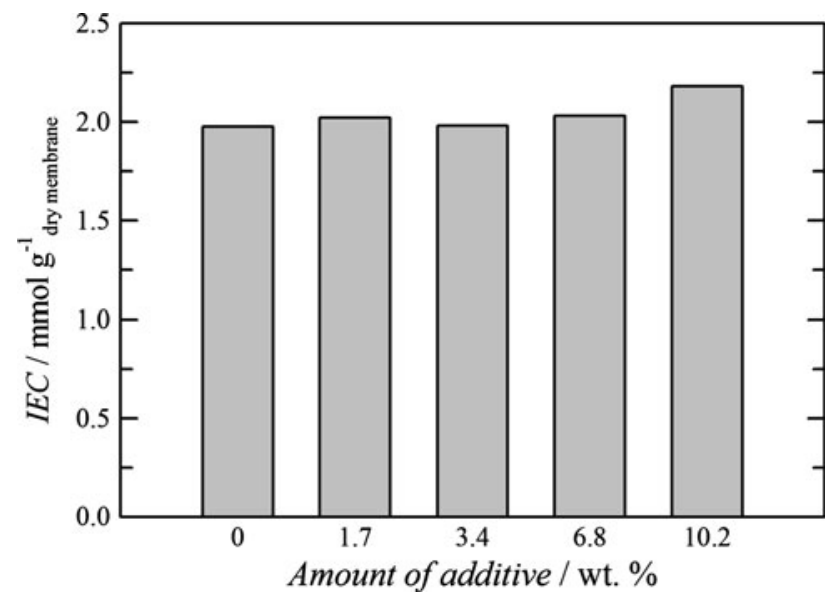

Fig. 5 Dependence of the apparent ion-exchange capacity on the amount of water-soluble component added to the structure of the membrane

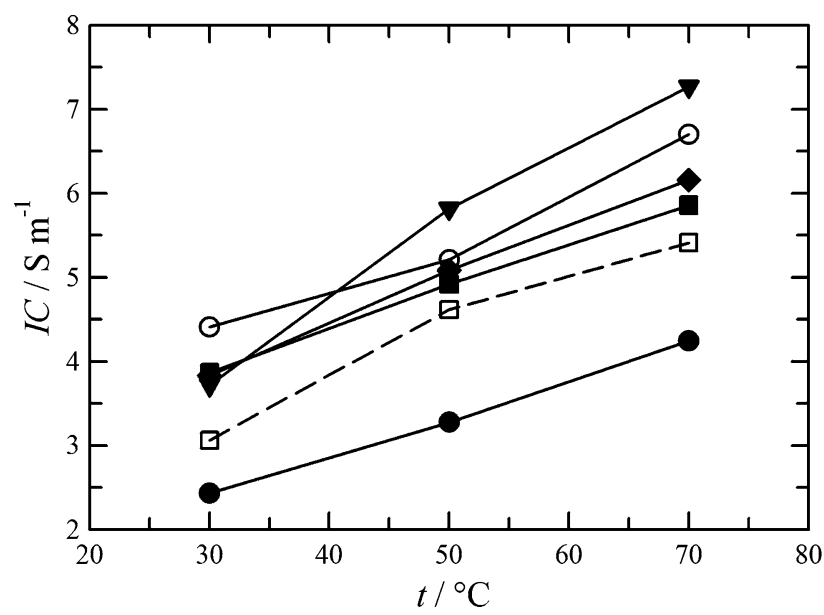

Fig. 6 Dependence of the ionic conductivity of the tested sample with different amounts of water-soluble additive on the temperature. $0 \mathrm{wt} \%$ of the additive-solid circle symbol; $1.7 \mathrm{wt} \%$ of the additivesquare symbol; $3.4 \mathrm{wt} \%$ of the additive-diamond symbol; $6.8 \mathrm{wt} \%$ of the additive-inverted triangle symbol; $10.2 \mathrm{wt} \%$ of the additiveopen circle symbol. Open square symbol-reference membrane A201 (Tokuyama, Japan)

either for heterogeneous or homogeneous anion-exchange membranes [16-18].

\subsection{Ionic conductivity}

The ionic conductivity of the membranes in dependence on temperature is shown in Fig. 6 for the samples with five different PEG-PPG contents together with the reference membrane. For all samples, the IC increases with the temperature. This has two reasons. The primary one is the decrease in the viscosity of the pore fluid with increasing temperature, and thus in higher mobility of the ions in the membrane. The second is connected with the mechanical

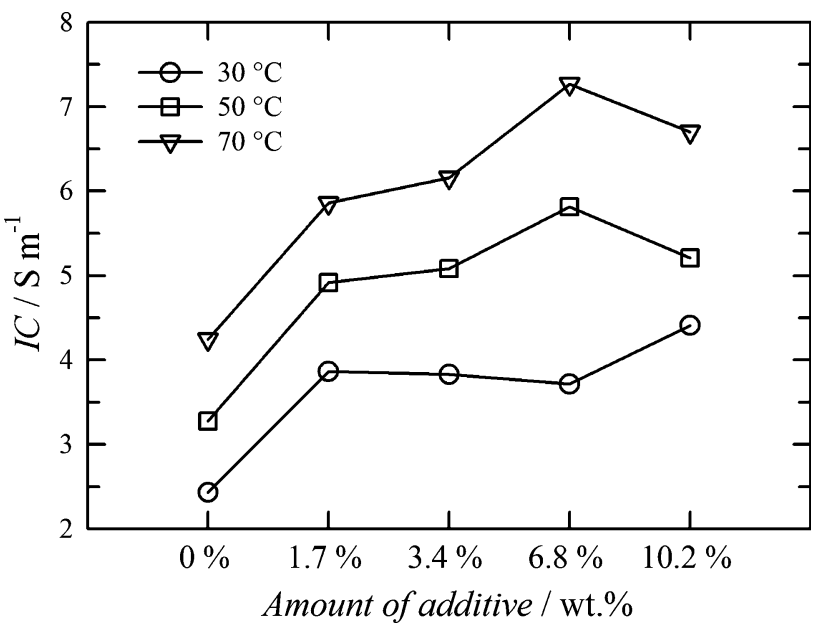

Fig. 7 Dependence of the ionic conductivity on the amount of the water-soluble component measured for three different temperatures. Temperatures are indicated in the top left-hand corner

properties of the membranes. With increasing temperature, the mechanical strength of the two polymer backbones decreases, thus allowing additional swelling of the anionexchange particles, which positively influences their conductivity.

In contrast to IEC, the IC is evidently positively influenced by the addition of PEG-PPG to the matrix polymer. The IC of all the membrane samples with water-soluble additive even exceeded that of the reference membrane A201. To illustrate IC dependence on the addition of PEGPPG more clearly, it is shown for three different temperatures in Fig. 7. It is evident that the most pronounced IC increase occurs with the first addition of PEG-PPG. The impact of further additive content is related to the operational temperature. Whereas at $30{ }^{\circ} \mathrm{C}$ no significant effect of the increased additive content was observed, at 50 and $70{ }^{\circ} \mathrm{C}$ the situation is different. Since, from the viewpoint of the intended application, elevated temperatures are especially of interest. The following discussion will mainly focus on them. At a temperature of $70{ }^{\circ} \mathrm{C}$, the highest ionic conductivity of $7.2 \mathrm{~S} \mathrm{~m}^{-1}$ was achieved in the case of the membrane with an addition of $6.8 \mathrm{wt} \%$ of the water-soluble component. This value is higher than the IC of many other heterogeneous $[17,19,20]$ or homogeneous $[18,21,22]$ anion-exchange membranes reported in the literature. A further increase in the PEG-PPG addition resulted in a decrease of the IC.

The reason for the difference between the behavior of IEC and IC consists in the character of these values. Whereas IEC represents an equilibrium parameter, as discussed in the previous chapter, IC is, in principle, a parameter limited by the mobility of the ions in the environment and by their concentration. As follows from the IEC experiments, in all samples the concentration of 
the charge carriers is constant within experimental error. Thus, their mobility is decisive. Increasing porosity of the surface skin layer as well as the polymer binder phase in the membrane interior most probably improve this aspect, thus resulting in enhanced membrane conductivity. The decrease observed for the membrane samples with the highest content of water-soluble additive may be related to the deteriorated mechanical properties of the membrane. Once the matrix polymer loses a decisive portion of its original mechanical strength, this allows a more significant opening of its structure. This leads to a loss of mechanical contact among a certain portion of the ion-exchange particles. As a consequence, the ionic conductivity decreases. This process is enhanced by the increasing temperature due to the related change of the mechanical strength of the LDPE with increasing temperature. Therefore, this reduction in conductivity was not observed at $30^{\circ} \mathrm{C}$. The rigidity of the matrix polymer at $30^{\circ} \mathrm{C}$ also most probably explains the fact that the IC value of the membrane was not observed to have been influenced by the higher content of the water-soluble addition in the matrix polymer.

\subsection{Alkaline water electrolysis}

Final testing of the membranes was performed in a laboratory alkaline water electrolyzer at a temperature of $70{ }^{\circ} \mathrm{C}$. The electrolysis load curves measured for the membrane samples tested are shown in Fig. 8. From the dependencies shown, it is possible to see a significant increase in process efficiency with the addition of PEGPPG up to $3.4 \mathrm{wt} \%(40 \%$ at $1.9 \mathrm{~V})$. A further increase to

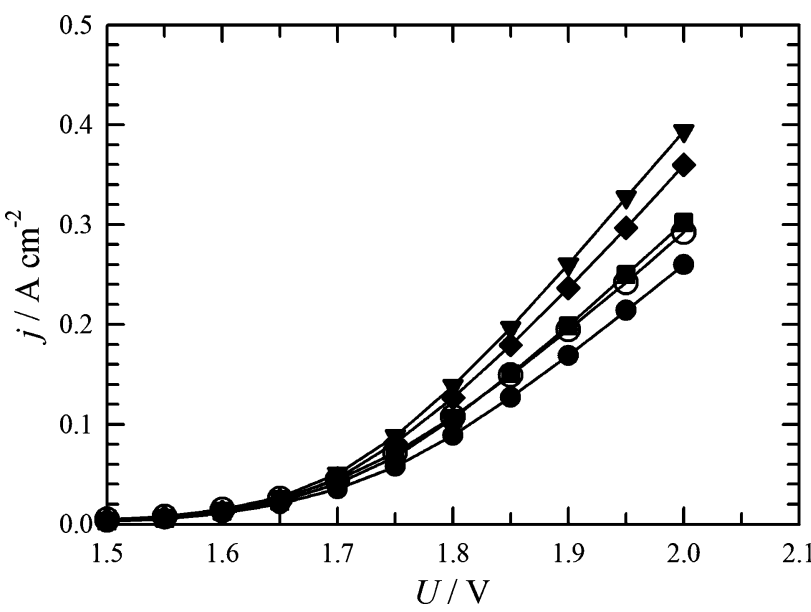

Fig. 8 Dependence of the current density measured on cell voltage. The measurement was made at a temperature of $70{ }^{\circ} \mathrm{C}$ in $10 \mathrm{wt} \%$ $\mathrm{KOH}$ solution with $\mathrm{Ni}$ foam used both as anode and cathode (geometrical area $4 \mathrm{~cm}^{2}$ ). $0 \mathrm{wt} \%$ of the additive-full circle symbol; $1.7 \mathrm{wt} \%$ of the additive-square symbol; $3.4 \mathrm{wt} \%$ of the additivediamond symbol; $6.8 \mathrm{wt} \%$ of the additive-inverted triangle symbol; $10.2 \mathrm{wt} \%$ of the additive-open circle symbol
$6.8 \mathrm{wt} \%$ only resulted in an insignificant improvement in the system performance ( $10 \%$ at $1.9 \mathrm{~V})$. It should be noted that, in the case of the membrane with $6.8 \mathrm{wt} \%$, a current load value of $0.26 \mathrm{~A} \mathrm{~cm}^{-2}$ achieved at $1.9 \mathrm{~V}$ is comparable with values achieved in industry despite the fact that in industry up to $30 \mathrm{wt} \%$ of potassium hydroxide is used [5]. With a $10.2 \mathrm{wt} \%$ of PEG-PPG, the load curve decreases due to the deterioration of the mechanical properties discussed earlier in this work.

To obtain greater insight into this behavior under conditions of alkaline water electrolysis, an analysis of the EIS was carried out and the corresponding Nyquist and Bode diagrams are shown in Figs. 9 and 10, respectively. It is possible to see that, in the frequency range of $10 \mathrm{kHz}-$ $100 \mathrm{~Hz}$ with a perturbation signal amplitude of $5 \mathrm{mV}$, there is only one semicircle visible in the Nyquist diagram for the voltage range studied of 1.5-1.9 V. One maximum, visible in the dependence of the phase shift shown in the Bode diagram, confirms the existence of just one kinetic rate constant in the system, or its dominance. This observation implies that one of the electrode's reactions plays the role of the rate-determining step in the system. With regard to the electrode materials used and the conditions of alkaline water electrolysis, it is possible to suspect that this electrode reaction is related to anodic oxygen evolution. On the basis of this discussion, a simple Randles equivalent circuit was used to evaluate the solution resistance in the cell. In this case, the RC element of this equivalent circuit

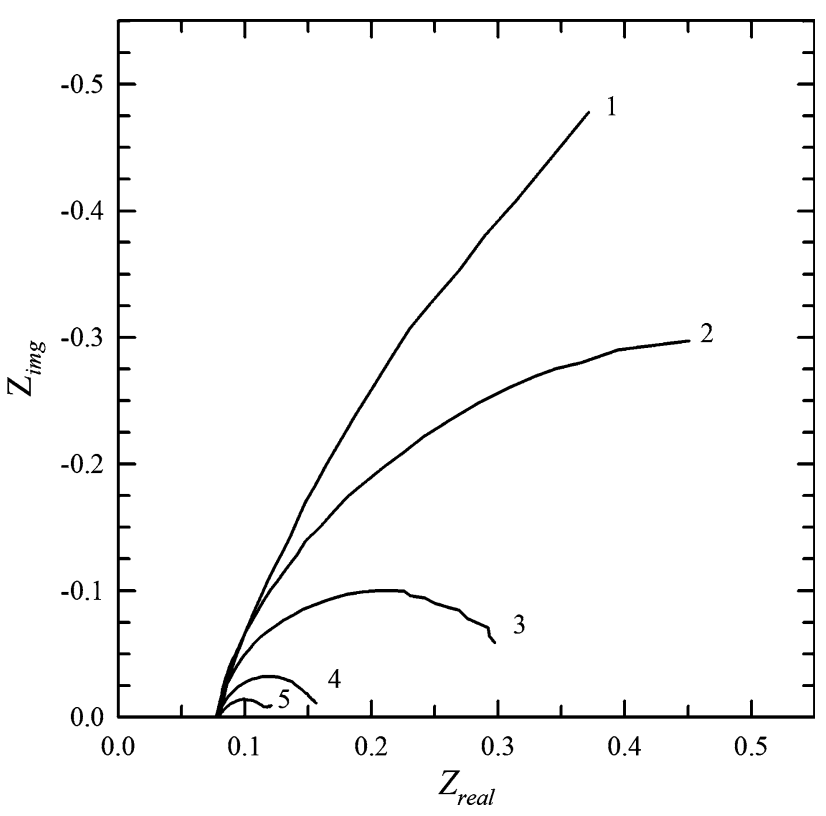

Fig. 9 Nyquist diagrams obtained by EIS under conditions of alkaline water electrolysis in a two-electrode arrangement, frequency range of $10 \mathrm{kHz}-100 \mathrm{~Hz}$, perturbation signal amplitude $5 \mathrm{mV}$, membrane with $6.8 \mathrm{wt} \%$ PEG-PPG addition. Line $11.5 \mathrm{~V}$, line 2 $1.6 \mathrm{~V}$, line $31.7 \mathrm{~V}$, line $41.8 \mathrm{~V}$, line $51.9 \mathrm{~V}$ 


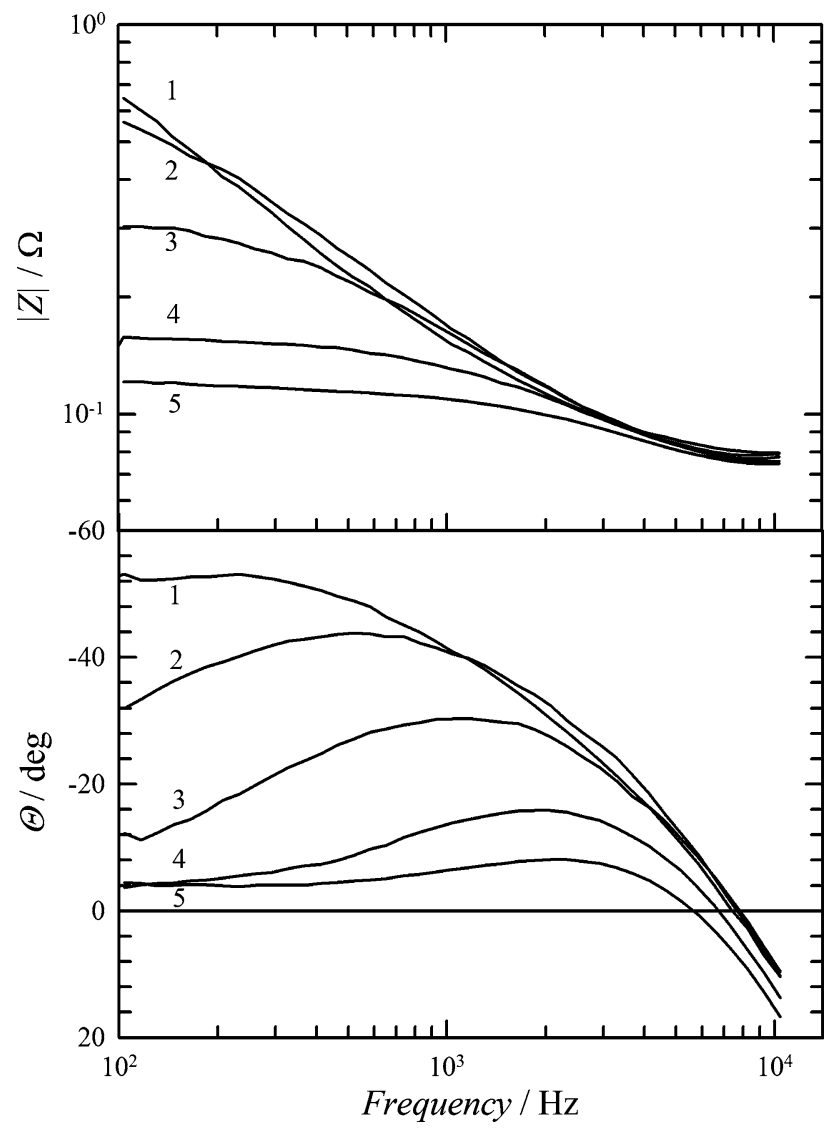

Fig. 10 Bode diagrams obtained by EIS under conditions of alkaline water electrolysis in a two-electrode arrangement. The range of the frequencies $10 \mathrm{kHz}-100 \mathrm{~Hz}$. Maximal amplitude $5 \mathrm{mV}$. A membrane with $6.8 \mathrm{wt} \%$ of PEG-PPG was used. Line $11.5 \mathrm{~V}$, line $21.6 \mathrm{~V}$, line $31.7 \mathrm{~V}$, line $41.8 \mathrm{~V}$, line $51.9 \mathrm{~V}$

corresponds to the anodic reaction. An ohmic resistance connected in series to this element then corresponds to the electrolyte, i.e., the anion-selective membrane used. Since electrode reaction kinetics is not the subject of this work, the results of the polarization resistance of the electrode reaction are not presented here. Attention focuses on the electrolyte resistance.

The dependencies of the individual membranes' resistance on the cell voltage are shown in Fig. 11. With regard to the fact that the concentration of the liquid electrolyte was always identical, any change in the membrane resistance detected is thus related to its properties. The highest IC under conditions of alkaline water electrolysis was achieved in the case of the membrane originally containing $6.8 \mathrm{wt} \%$ of water-soluble additive. These results are thus consistent with the IC data as well as with the load curves under conditions of alkaline water electrolysis. The fact that the membrane resistance is independent of the cell voltage proves the correctness of the experimental arrangement. A change in the membrane resistance with the changing voltage would indicate penetration of the gas

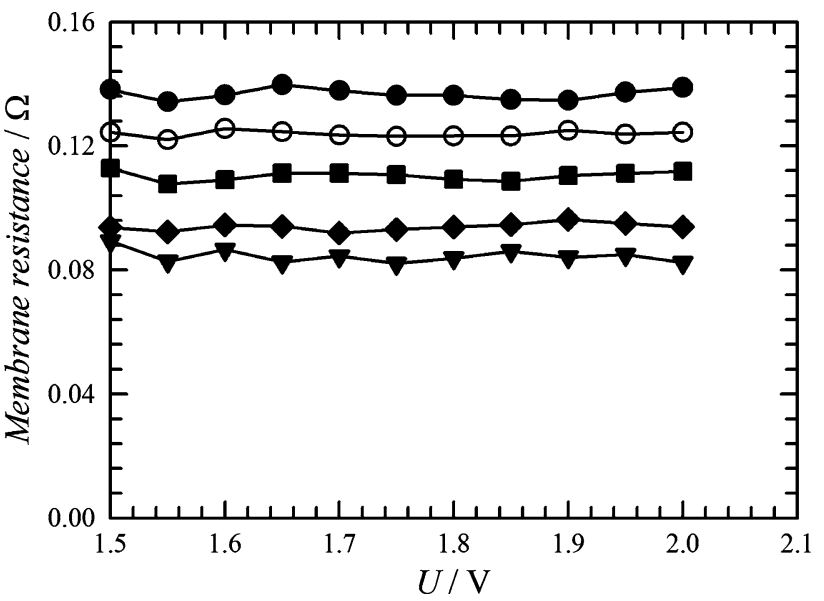

Fig. 11 Dependence of membrane resistance on the water electrolysis cell voltage, temperature $70^{\circ} \mathrm{C}, 10 \mathrm{wt} \% \mathrm{KOH}$ solution, $\mathrm{Ni}$ foam used both as anode and cathode (geometrical area of $4 \mathrm{~cm}^{2}$ ). 0 wt $\%$ of the additive-solid circle symbol; $1.7 \mathrm{wt} \%$ of the additivesquare symbol; $3.4 \mathrm{wt} \%$ of the additive-diamond symbol; $6.8 \mathrm{wt} \%$ of the additive-inverted triangle symbol; $10.2 \mathrm{wt} \%$ of the additiveopen circle symbol

phase into the space between the electrodes. This would mean that the electrodes were not in direct contact with the membrane. Such a result would not be reliable with respect to an evaluation of the membrane properties.

\section{Conclusion}

A series of heterogeneous anion-selective membranes with increased porosity of the surface skin was prepared and tested from the point of view of their mechanical and electrochemical properties. The addition of different amounts of a water-soluble additive was used to increase the porosity of the surface skin and consequently the electrochemical properties of the membrane, mainly the ionic conductivity. This was achieved at the expense of the resulting mechanical stability of the membrane. However, deterioration of this factor did not reach a critical level from the point of view of the reliability of the electrolysis cell. The positive effect of the increased porosity of the surface skin on the membrane properties was also proven in the laboratory water electrolysis cell.

The development of an anion-selective polymer electrolyte with satisfactory properties represents a necessary prerequisite for further development of the alkaline water electrolysis process. The connection of a stable and conductive polymer electrolyte with a corresponding binder will allow a membrane electrode assembly similar to the acidic process to be produced and thus to significantly improve the intensity and flexibility of the process. 
Acknowledgments Financial support of this work by the Ministry of Industry and Trade of the Czech Republic within the framework of project No. FR-TI2/442, by the WELTEMP Project, Contract No. 212903 and by the Ministry of Education, Youth and Sports of the Czech Republic within the framework of the Specific University Research, Project No. MSMT No. 21/2011, is gratefully acknowledged.

\section{References}

1. Hnát J, Paidar M, Schauer J et al (2011) J Appl Electrochem 41:1043

2. European Photovoltaic Industry Association (2010) Market Outlook 2010. http://www.epia.org/fileadmin/EPIA_docs/documents/ press/Market_Outlook_2010.pdf. Accessed 6 Jan 2012

3. Frano B (2005) Sol Energy 78:661

4. Holladay JD, Hu J, King DL et al (2009) Catal Today 139:244

5. Häussinger P, Lohmüller R, Watson AM (2000) Hydrogen, 2. Production, Wiley-VCH Verlag GmbH \& Co. KGaA. http://dx. doi.org/10.1002/14356007.o13_o03. Accessed 28 Nov 2011

6. Zeng K, Zhang D (2010) Prog Energy Combust Sci 36:307

7. Varcoe JR, Slade RCT (2005) Fuel Cells 5:187

8. Jang BZ, Uhlmann DR, Sande JBV (1984) J Appl Polym Sci 29:4377
9. Liang JZ, Li RKY (2000) J Appl Polym Sci 77:409

10. Pukánszky B, Móczó J (2004) Macromol Symp 214:115

11. ExxonMobil Chemicals (2012) ExxonMobil ${ }^{\mathrm{TM}}$ LDPE LD 605BA low density polyethylene resin. http://exxonmobilchemical.ides. com/datasheet.aspx? $\mathrm{I}=58933 \& \mathrm{PS}=\mathrm{COMBO} \& \mathrm{E}=51275$. Accessed 9 Jan 2012

12. EXACT 0210, (2012) Dexplastomers. http://www.dexplastomers. com/products/exact/grade/_down/Exact_0210_V02_2011.pdf. Accessed 14 May 2012

13. Plastic international (2011) LDPE datasheet. http://www.plastic sintl.com/datasheets/LDPE.pdf. Accessed 28 Nov 2011

14. Yu EH, Krewer U, Scott K (2010) Energies 3:1499

15. Bouzek K, Moravcová S, Schauer J et al (2010) J Appl Electrochem 40:1005

16. Lebrun L, Follain N, Metayer M (2004) Electrochim Acta 50:985

17. Merle G, Wessling M, Nijmeijer K (2011) J Membr Sci 377:1

18. Wang G, Weng Y, Chu D et al (2009) J Membr Sci 326:4

19. Vassal N, Salmon E, Fauvarque JF (2000) Electrochim Acta 45:1527

20. Lewandowski A, Skorupska K, Malinska J (2000) Solid State Ion 133:265

21. Zeng QH, Liu QL, Broadwell I et al (2010) J Membr Sci 349:237

22. Stoica D, Ogier L, Akrour L et al (2007) Electrochim Acta 53:1596 